\title{
Web-based, rapid and contactless management of ambulatory patients for SARS-CoV-2-testing
}

Jannik Stemler

University Hospital of Cologne https://orcid.org/0000-0001-9152-2469

\section{Oliver A. Cornely}

University Hospital of Cologne https://orcid.org/0000-0001-9599-3137

Torsten Noack-Schönborn

University Hospital of Cologne

\section{Corinna Forholz}

Healex $\mathrm{GmbH}$

\section{Sofie Schumacher}

University Hospital of Cologne https://orcid.org/0000-0002-8805-1421

Leonard Poluschkin

University Hospital of Cologne https://orcid.org/0000-0002-2727-2765

\section{Bernd Binder}

University Hospital of Cologne

\section{Clara Lehmann}

University Hospital of Cologne

Georg Langebartels ( $\nabla$ georg.langebartels@uk-koeln.de )

University Hospital of Cologne

\section{Research Article}

Keywords: COVID-19 pandemic, SARS-CoV-2 testing strategy, web-based patient management, digital medicine

Posted Date: November 25th, 2020

DOI: https://doi.org/10.21203/rs.3.rs-114537/v1

License: (c) (i) This work is licensed under a Creative Commons Attribution 4.0 International License. Read Full License

Version of Record: A version of this preprint was published at Infection on November 25th, 2020. See the published version at https://doi.org/10.1007/s15010-021-01716-4. 


\section{Abstract}

\section{Background}

During the SARS-CoV-2 pandemic a mass casualty incident of ambulatory patients occurred at the COVID-19 rapid response infrastructure (CRRI) facility at the University Hospital of Cologne (UHC). We report the development of a patient-centred mobile-device solution to support efficient management of the facility, triage of patients and rapid delivery of test results.

\section{Methods}

The UHC-Corona Web Tool (CWT) was developed as a web-based application useable on each patient's smartphone. It provides, among others, a self-reported medical history including type and duration of symptoms and potential risk contacts and links all retrieved information to the digital patient chart via a QR code. It provides scheduling of outpatient appointments and automated transmission of SARS-CoV-2 test results.

\section{Results}

The UHC-CWT was launched on April $9^{\text {th }}, 2020$. It was used by 28652 patients until August $31^{\text {st }}, 2020$. Of those, 15245 $(53,2 \%)$ consulted the CRRI, representing $43,1 \%$ of all CRRI patients during the observed period.

There were $8304(29,0 \%)$ specifications concerning travel history and $17145(59,8 \%)$ indications of $\geq 1$ symptom of SARS-CoV-2 infection. The most frequently indicated symptoms were sore throat (60,0\%), headache $(50,7 \%)$, common cold $(45,1 \%)$ and cough $(42,6 \%)$ while $11057(40,2 \%)$ patients did not report any symptoms. After implementation of the UHC-CWT, the number of patient contacts per physician rose from 38 to 98,7 per day. The personnel for communication of test results was reduced from four on seven days to one on five days.

\section{Conclusion}

The UHC-CWT is an effective digital solution for management of large numbers of outpatients for SARS-CoV-2 testing.

\section{Background}

From the end of February 2020 onwards, a large number of patients were seen at the emergency department as well as the COVID-19 rapid response infrastructure (CRRI) facility of the University Hospital of Cologne (UHC) in context of the SARS-CoV-2 pandemic (1). Extensive prior preparation was not possible. The close geographical proximity to one of Germany's first SARS-CoV-2 hotspots in the Heinsberg area on top of numerous travel returners from the Alpine region, led to maxed-out capacity $(2,3)$. In addition, a large number of UHC employees with a relevant travel history or contact with SARS-CoV-2 positive patients had to be tested to avoid further spread of the disease within the hospital system (4, 5).

This mass influx of ambulatory patients demanded a drastic increase of efficiency up-front triage of patients, consultations and rapid communication of test results while ascertaining constant quality.

In order to reduce consultation time to a reasonable minimum, a paper-based questionnaire on prior medical history was distributed to waiting patients. The questionnaire was available in several languages and updated on a daily basis to include new findings, such as newly declared high-risk areas or newly identified symptoms of COVID-19 such as 
olfactory or taste disorders (6-8). This questionnaire was then scanned and included as source data in the individual electronic patient file.

It soon became evident, that a digital approach was needed to support this overwhelming situation.

\section{Methods}

The following goals were set: enabling patients to take their own medical history, embedding the Web Tool into the IT system of the CRRI and the automated communication of negative SARS-CoV-2-PCR results.

\section{Development of the UHC Corona Web Tool}

The rapidly changing epidemiological situation required a continuously flexible software development in a limited time frame. Definition of the process flow and legal review of applicable data security and privacy requirements as well as development of texts for automated electronic notifications took place simultaneously. The development of the UHC Corona Web Tool (Healex GmbH, Cologne, Germany) was conducted in accordance with principles of agile software development using the Scrum method. The Scrum method entails the setting of a time frame (so-called Sprint), in this case two weeks, in which the product increment is developed. In preparation of the Sprint, requirements are defined and prioritized. In the course of the Sprint, a continuous exchange between development team and users takes place. By the end of the Sprint, as many defined requirements as possible should be met and the product should be finalized.

The electronic patient chart regularly used by UHC in the clinical information system (CIS) ORBIS $\circledast$ (Dedalus HealthCare $\mathrm{GmbH}$, Bonn, Germany) and SAP-SE (SAP, Walldorf, Germany) were linked with the patient chart of the UHC Corona Web Tool.

\section{Utilization of the UHC Corona Web Tool}

The UHC Corona Web Tool is announced via internet, signage on the hospital campus as well as the mobile CRRI. A smartphone call to the UHC hotline initiates automatically a SMS with a link being sent to the caller. The link opens the UHC Corona Web Tool on the smartphone browser. Once the patient has agreed to the data privacy terms, he/she can provide his/her personal medical history (Fig. A1). The Web Tool then sends the following information to the caller: 1) travel directions to the CRRI, 2) a link to schedule an appointment 3) a link including a QR-code. At the CRRI site, the QRcode is scanned to transfer the patient-provided medical history to the electronic patient chart in the CIS. (Fig. 1)

All features can be used via any standard internet browser on an ordinary smartphone and do not require installation of an additional app.

\section{Self-reported medical history and test implementation}

The self-reported medical history conducted through the UHC Corona Web Tool records the type and duration of symptoms, risk contacts, stays in high risk-areas, underlying diseases and prognostic factors. The questionnaire can be answered before entering the CRRI.

During the subsequent physician consultation, the indication for SARS-CoV-2 testing via nasopharyngeal swab is evaluated. Current recommendations of the German Centre for Disease Control, the Robert Koch Institute (RKI) are applied, whilst taking into account further epidemiological and clinical criteria $(9,10)$.

A negative SARS-CoV-2-PCR test result is communicated within seconds via an automatically dispatched SMS right after validation of the PCR test result by a UHC virologist. A data link between the virologic laboratory and the UHC 
Corona Web Tool allows for this notification. Positive test results are communicated via a telephone call consultation conducted by a physician (Fig. 1). Additionally, a link to a fourteen-day symptom entry log is sent to the caller in order to document the potential development of symptoms suggestive of COVID-19. This also includes a calendar notification function. The log is developed in accordance with recommendations of the RKI (11).

\section{Data privacy and ethical statement}

The application of the UHC Corona Web Tool does not require an external login into a patient file, nor does it require access to a hospital's IT system. All user interaction is conducted via SMS using the individual's phone number. No personal information is shared or made available online at any given time. According to the technical state of the art, compromise of the data through third parties is therefore not possible. The consent to use the anonymized data is actively given by the user. A revocation of consent is possible via the data safety officer of the UHC. The data is completely anonymized before analysis therefore no specific ethical statement was obtained.

\section{Analysis}

The data generated through the implementation of the UHC Corona Web Tool concerning symptoms, risk contacts and SARS-CoV-2 test results was analysed in a descriptive manner. A possible influence of the increased usage of the UHC Corona Web Tool on efficiency in the consultation was assessed by quantitatively analysing the patient contact per physician. The cut-off date of the here presented data is August, $31^{\text {st }} 2020$.

\section{Results}

The first operational application of the UHC Corona Web Tool was completed in one sprint. In a following internal test phase, further requirements were evaluated. After three weeks of development, the application was launched on the April, $9^{\text {th }}$ 2020. Since project initiation, the methodology for further development has remained the same. This method entails an update conducted every two weeks regarding additional user functions as well as updates necessitated by the epidemiological situation.

Geographically, the CRRI's catchment area includes the adjacent neighbourhoods as well as other areas within and around the city of Cologne, Germany. The number of patients per region (postal code and country) can be found in Fig. A2a-d.

Since the establishment of the CRRI, a total of 35378 ambulatory patients were seen until the 31 st of August 2020 and 36214 nasopharyngeal swabs for SARS-CoV-2-PCR tests were conducted in this time period. The discrepancy in results is due to multiple testing of some individual patients. On average, 1263 patients per week over the described times period were seen. Prior to the implementation of the UHC Corona Web Tool 1150 patients per week were seen. After implementation of the UHC Corona Web Tool, 27515 patients were seen in the CRRI (1310 per week on average) (Tab. 1).

The UHC Corona Web Tool was used by 28652 patients. Of these, 17145 users (59.8\%) filled out the questionnaire on medical history completely. This resulted in 8304 (29.0\%) data entries on travel information and 487 (1.7\%) data entries on potential risk contacts with SARS-CoV-2 positive persons. The latter entry option was excluded from the 27 th calendar week onwards due to users' inaccurate data entry and additional potential confounders. Overall, 17145 (59.8\%) of patients reported symptoms. The most common symptoms were: sore throat (60.0\%), headache (50.7\%), common flu-like symptoms (45.1\%) and cough (42.6\%). The remaining 11057 patients (40.2\%) reported no symptoms at all (Fig. 2). 
15245 users of the of the UHC Corona Web Tool (53.2\%) visited the CRRI in person. Of these, 12228 patients (80.3\%) underwent SARS-CoV-2-PCR testing via nasopharyngeal swab. 52 tests $(0.43 \%)$ were positive and 12172 were negative for SARS-CoV-2 (Fig. 2).

Prior to the implementation of the UHC Corona Web Tool, the average number of patient contacts per physician per day was 38 with a maximum of 59 patient contacts per physician per week in the $12^{\text {th }}$ calendar week (Tab. 1). After introduction of the UHC Corona Web Tool, the total number of patient contacts in the CRRI per week initially decreased. Also, the number of employed physicians at the CRRI decreased and the opening hours were reduced from seven days to five days a week. In the further course, the number of patient contacts in the CRRI increased, partially due to a public call for intensified testing (Fig. 3). Subsequently, the number of patient contacts per physician per day increased to 98.7 with a maximum of 893 patients per physician per week in the $35^{\text {th }}$ calendar week (Fig. 3 and Tab. 1).

The communication of test results through the UHC SARS-CoV-2 contact management team was optimized. After introduction of the UHC Corona Web Tool, the personnel required was reduced from four employees in two shifts on seven days to one employee available during a regular working day on five days per week.

\section{Discussion}

The efficient deployment of healthcare personnel plays a decisive role in managing a critical outbreak during the SARSCoV-2 pandemic (12). Our study highlights the use of a web-based patient management at a COVID-19 testing facility. The introduction of the UHC Corona Web Tool supported SARS-CoV-2 testing by increasing patient contacts per physician. Furthermore, the personnel required for the communication of test results was reduced. We report the methodology of an easy-to-use and accessible solution in order to facilitate triage, testing and result delivery for SARSCoV-2 testing. We report findings from an analysis of the user data of 28652 users increasing efficiency from an average patient number per physician per day from 38 up to 98.7 .

A variety of apps have been developed to manage the SARS-CoV-2 pandemic (13). However, app development is lengthy, depending on the used platform and has a range data of regulatory pitfalls. The main difference between apps and our web-based method is the personalized link which identifies the patient just in that single case of admittance. By using a web-based solution we were able to establish a satisfactory process very quickly while meeting all applicable data protection requirements. As a web-based tool, no additional application needs to be installed. The tool works via a browser and is compatible with almost every device. Web-based approaches to record symptoms and subsequent medical implications in the context of COVID-19 have been developed and validated by other groups (14). Our approach includes a patient-centred perspective and additionally connects it with a number of other functions.

The UHC Corona Web Tool enables triage of patients via symptom analysis in the following manner: 1.) Patients with contact to a SARS-CoV-2 positive tested person 2.) symptomatic and 3.) asymptomatic patients. This triage is carried out before the patient enters the CRRI. This procedure prevents possible transmissions of the virus in the CRRI site itself $(15,16)$. In addition, the contactless use of smartphones prevents possible infections via touch of surfaces and provided materials such as pens as well as close contact while exchanging these items $(17,18)$. Mass testing sites are known to play a key role in the control of pandemics (19). By connecting the UHC Corona Web Tool to the scheduling of appointments, the CRRI capacity can be optimized. This also facilitates the allocation of required testing materials.

By using a web-based approach, time pressure on patients reporting their medical history is avoided which can increase accuracy and validity of the medical history. Through storage of the fourteen-day-long symptoms log on the patient's smartphone, the documentation of the development of symptoms is easily accessible and may help to facilitate contact tracing by public healthcare institutions. In line with other research, the most common symptoms suggestive of SARS- 
CoV-2 infection in the described cohort were: common cold, cough, headache and sore throat (20). According to the current state of knowledge, these symptoms have the highest sensitivity concerning mild and ambulatory SARS-CoV-2 infections. These symptoms, however, are unspecific and are of low diagnostic relevance (21). Loss of taste and smell was included after launching the Web Tool, therefore, this symptom is not adequately represented in our analysis (22, 23).

The percentage of UHC Web Tool users who subsequently underwent SARS-CoV-2 testing was low in comparison to all patients consulted at the CRRI. Especially UHC employees received multiple tests, which led to an increase in the overall rate of tests. These employees, however, did not use the UHC Corona Web Tool as often. It is possible, that the increase in efficiency is due to stricter evaluation regarding test indications updated by the RKI as well as due to the implementation of the UHC Corona Web Tool (9).

Our approach certainly has limitations. The usage of the UHC Corona Web Tool is voluntary. Since older patients do not use smartphones as frequently, the population that uses the UHC Corona Web Tool differs from the groups at risk of severe SARS-CoV-2 infection (24). The UHC Corona Web Tool is continuously updated due to the dynamic of the COVID19 pandemic. This may limit the analysis of the data. The here described data from mid-April to August represents a low incidence period of SARS-CoV-2 in Germany. Lack of internet connection and other technical difficulties could cause lack of adherence.

Applications like the UHC Corona Web Tool can provide detailed and anonymized data for analysis of the development of infections in a scientific context(25). The utilization of a web-based application provides new perspectives for the future of the healthcare system in general and specifically with regard to future pandemics regardless of their aetiology. Add-ons such as automated timely reporting to public healthcare institutions in accordance with any legislation could be implemented (26). This might further decrease work load and optimize personnel allocation during a critical pandemic situation in the near future. A web-based approach can be part of an emergency strategy for mass testing or part of routine testing in order to avoid regional lockdowns and thus prevent substantial disruptions in everyone's daily life. An immediate communication of test results is possible. Therefore, critical time delays in regard to the identification of infection chains are no longer encountered (27).

\section{Conclusions}

The UHC Corona Web Tool facilitated SARS-CoV-2 testing by increasing patient contacts per physician. Also, the personnel required to communicate test results was reduced. Applications like the UHC Corona Web Tool can support the collection of data to analyse developments in infectious diseases. Web-based applications open up new perspectives for our future healthcare systems, especially in regard to future pandemics. The UHC Corona Web Tool represents an effective digital solution for the overall management from admission right through to the test result for large scale testing of ambulatory patients during the COVID-19 pandemic.

\section{Declarations}

Ethics approval: not applicable

Consent to participate: all patients agreed to anonymized scientific evaluation of their reported data via the UHC WebTool

Consent for publication: not applicable 
Availability of data and material: All data, materials and software application comply with field standards regarding data transparency. The datasets analyzed are available from the corresponding author upon reasonable request.

Competing interests: JS reports research grants from Basilea Pharmaceuticals Inc., and travel grants from MetaAlexander Foundation and from German Society for Infectious Diseases e.V. outside the submitted work; OAC is supported by the German Federal Ministry of Research and Education, is funded by the Deutsche Forschungsgemeinschaft (DFG, German Research Foundation) under Germany's Excellence Strategy - CECAD, EXC 2030 - 390661388 and has received research grants from Actelion, Amplyx, Astellas, Basilea, Cidara, Da Volterra, F2G, Gilead, Janssen, Medicines Company, Melinta, Merck/MSD, Octapharma, Pfizer, Scynexis, is a consultant to Actelion, Allecra, Amplyx, Astellas, Basilea, Biosys, Cidara, Da Volterra, Entasis, F2G, Gilead, Matinas, MedPace, Menarini, Merck/MSD, Mylan, Nabriva, Noxxon, Octapharma, Paratek, Pfizer, PSI, Roche Diagnostics, Scynexis, and Shionogi, and received lecture honoraria from Al-Jazeera Pharmaceuticals, Astellas, Basilea, Gilead, Grupo Biotoscana, Merck/MSD and Pfizer outside the submitted work; TN-S declares no conflict of interest; CF is an employee of Healex $\mathrm{GmbH}$ and otherwise declares no conflict of interest; SS declares no conflict of interest; LP declares no conflict of interest; BB declares no conflict of interest; CL declares no conflict of interest; GL declares no conflict of interest.

Funding: The development of the UHC Corona Web Tool was funded by the University Hospital of Cologne Department for Clinical affairs and Crisis management. The analysis and preparation of this manuscript was carried out as part of our routine duties. No additional public or private funding was received for realization of this project.

Authors' contributions: GL, TNS, CF, BB, OAC and JS were part of the UHC Corona Web Tool development team. JS, TNS and OAC collected, analysed and interpreted data. All authors were involved in patient care or administration of the UHC CRRI. JS and OAC developed the first draft outline and wrote parts of the manuscript. All authors reviewed and approved the final manuscript.

\section{Acknowledgments}

We thank Stefan Wiesner, Healex $\mathrm{GmbH}$, for realization of the project. We thank M. Weber, A. Bensaid and N. Brück, from the UHC IT department for technical support. We thank Ullrich Bethe for critically reading and commenting the manuscript. We thank Susann Blossfeld for technical support. We thank all the nurses, doctors and staff of the University Hospital of Cologne and especially the CRRI for their strong attitude and continuous work during the pandemic.

\section{References}

1. Augustin M, Schommers P, Suarez I, Koehler P, Gruell H, Klein F, et al. Rapid response infrastructure for pandemic preparedness in a tertiary care hospital: lessons learned from the COVID-19 outbreak in Cologne, Germany, February to March 2020. Euro Surveill. 2020;25(21).

2. Laufs J, Elsbernd H, Hölzl F, Winter KD. [Coronavirus (SARS-CoV-2) - Outbreak in North Rhine-Westphalia (District of Heinsberg) - First diagnosis of a COVID-19 manifestation in a couple]. Dtsch Med Wochenschr. 2020;145(10):665-9.

3. Kreidl P, Schmid D, Maritschnik S, Richter L, Borena W, Genger JW, et al. Emergence of coronavirus disease 2019 (COVID-19) in Austria. Wien Klin Wochenschr. 2020:1-8.

4. Wu Z, McGoogan JM. Characteristics of and Important Lessons From the Coronavirus Disease 2019 (COVID-19) Outbreak in China: Summary of a Report of 72314 Cases From the Chinese Center for Disease Control and Prevention. Jama. 2020.

5. Ferioli M, Cisternino C, Leo V, Pisani L, Palange P, Nava S. Protecting healthcare workers from SARS-CoV-2 infection: practical indications. Eur Respir Rev. 2020;29(155).

Page $7 / 11$ 
6. Menni C, Valdes AM, Freidin MB, Sudre CH, Nguyen LH, Drew DA, et al. Real-time tracking of self-reported symptoms to predict potential COVID-19. Nat Med. 2020.

7. Mao L, Jin H, Wang M, Hu Y, Chen S, He Q, et al. Neurologic Manifestations of Hospitalized Patients With Coronavirus Disease 2019 in Wuhan, China. JAMA Neurol. 2020.

8. Täglicher Lagebericht des RKI zur Coronavirus-Krankheit-2019 (COVID-19) 31.03.2020 [press release]. Berlin: Robert Koch-Institut, 31-Mar-2020 2020.

9. COVID-19 Verdacht: Testkriterien und Maßnahmen. Robert Koch-Institut; 12 May 2020.

10. COVID-19: Verdachtsabklärung und Maßnahmen. Robert Koch-Institut; 26 Feb 2020.

11. Kurzfragebogen bzw. Tagebuch für Personen mit Kontakt zu COVID-19-Fällen Robert Koch-Institut; 17 Feb 2020 [Available from:

https://www.rki.de/DE/Content/InfAZ/N/Neuartiges_Coronavirus/Kontaktperson/Tagebuch_Kontaktpersonen.html.

12. Chen T, Wang Y, Hua L. "Pairing assistance": the effective way to solve the breakdown of health services system caused by COVID-19 pandemic. Int J Equity Health. 2020;19(1):68.

13. Bundesregierung D. Pressemitteilung - Unterstützt uns im Kampf gegen Corona Berlin: Die Bundesregierung; 2020 [Available from: https://www.bundesregierung.de/breg-de/themen/corona-warn-app.

14. Schrager JD, Schuler K, Isakov AP, Wright DW, Yaffee AQ, Jacobson KL, et al. Development and Usability Testing of a Web-based COVID-19 Self-triage Platform. West J Emerg Med. 2020;21(5):1054-8.

15. Kohanski MA, Lo LJ, Waring MS. Review of indoor aerosol generation, transport, and control in the context of COVID-19. Int Forum Allergy Rhinol. 2020.

16. Razzini K, Castrica M, Menchetti L, Maggi L, Negroni L, Orfeo NV, et al. SARS-CoV-2 RNA detection in the air and on surfaces in the COVID-19 ward of a hospital in Milan, Italy. Sci Total Environ. 2020;742:140540.

17. Otter JA, Donskey C, Yezli S, Douthwaite S, Goldenberg SD, Weber DJ. Transmission of SARS and MERS coronaviruses and influenza virus in healthcare settings: the possible role of dry surface contamination. J Hosp Infect. 2016;92(3):235-50.

18. Guo ZD, Wang ZY, Zhang SF, Li X, Li L, Li C, et al. Aerosol and Surface Distribution of Severe Acute Respiratory Syndrome Coronavirus 2 in Hospital Wards, Wuhan, China, 2020. Emerg Infect Dis. 2020;26(7):1583-91.

19. Peto J. Covid-19 mass testing facilities could end the epidemic rapidly. Bmj. 2020;368:m1163.

20. Tenforde MW, Billig Rose E, Lindsell CJ, Shapiro NI, Files DC, Gibbs KW, et al. Characteristics of Adult Outpatients and Inpatients with COVID-19 - 11 Academic Medical Centers, United States, March-May 2020. MMWR Morb Mortal Wkly Rep. 2020;69(26):841-6.

21. Struyf T, Deeks JJ, Dinnes J, Takwoingi Y, Davenport C, Leeflang MM, et al. Signs and symptoms to determine if a patient presenting in primary care or hospital outpatient settings has COVID-19 disease. Cochrane Database Syst Rev. 2020;7:Cd013665.

22. Chen X, Laurent S, Onur OA, Kleineberg NN, Fink GR, Schweitzer F, et al. A systematic review of neurological symptoms and complications of COVID-19. J Neurol. 2020:1-11.

23. Spinato G, Fabbris C, Polesel J, Cazzador D, Borsetto D, Hopkins C, et al. Alterations in Smell or Taste in Mildly Symptomatic Outpatients With SARS-CoV-2 Infection. Jama. 2020;323(20):2089-90.

24. Zhou F, Yu T, Du R, Fan G, Liu Y, Liu Z, et al. Clinical course and risk factors for mortality of adult inpatients with COVID-19 in Wuhan, China: a retrospective cohort study. Lancet. 2020;395(10229):1054-62.

25. Drew DA, Nguyen LH, Steves CJ, Menni C, Freydin M, Varsavsky T, et al. Rapid implementation of mobile technology for real-time epidemiology of COVID-19. Science. 2020. 
26. Leitmeyer KC, Espinosa L, Broberg EK, Struelens MJ, members tENFPle-rsg. Automated digital reporting of clinical laboratory information to national public health surveillance systems, results of a EU/EEA survey, 2018. Eurosurveillance. 2020;25(39):1900591.

27. tagesschau.de. Corona-Panne: Warten auf Testergebnisse aus Bayern tagesschau.de: NDR/ARD-aktuell; 2020 [updated 13-ß8-2020. Available from: https://www.tagesschau.de/inland/corona-soeder-test-103.html.

\section{Figures}

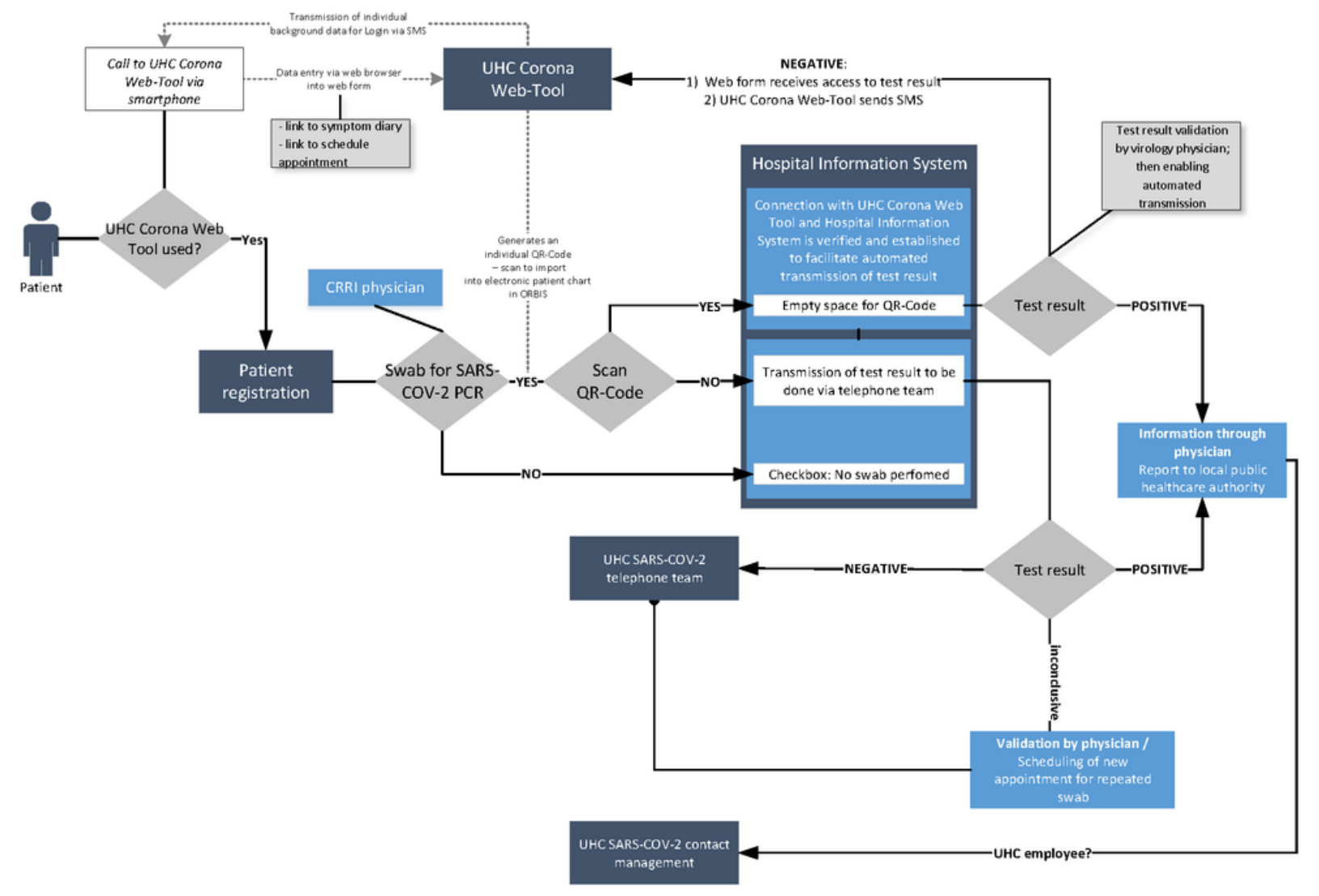

Figure 1

Flow diagram of the organizational structure of the CRRI including UHC Corona Web Tool CRRI, COVID-19 rapid response infrastructure; UHC, University Hospital Cologne 


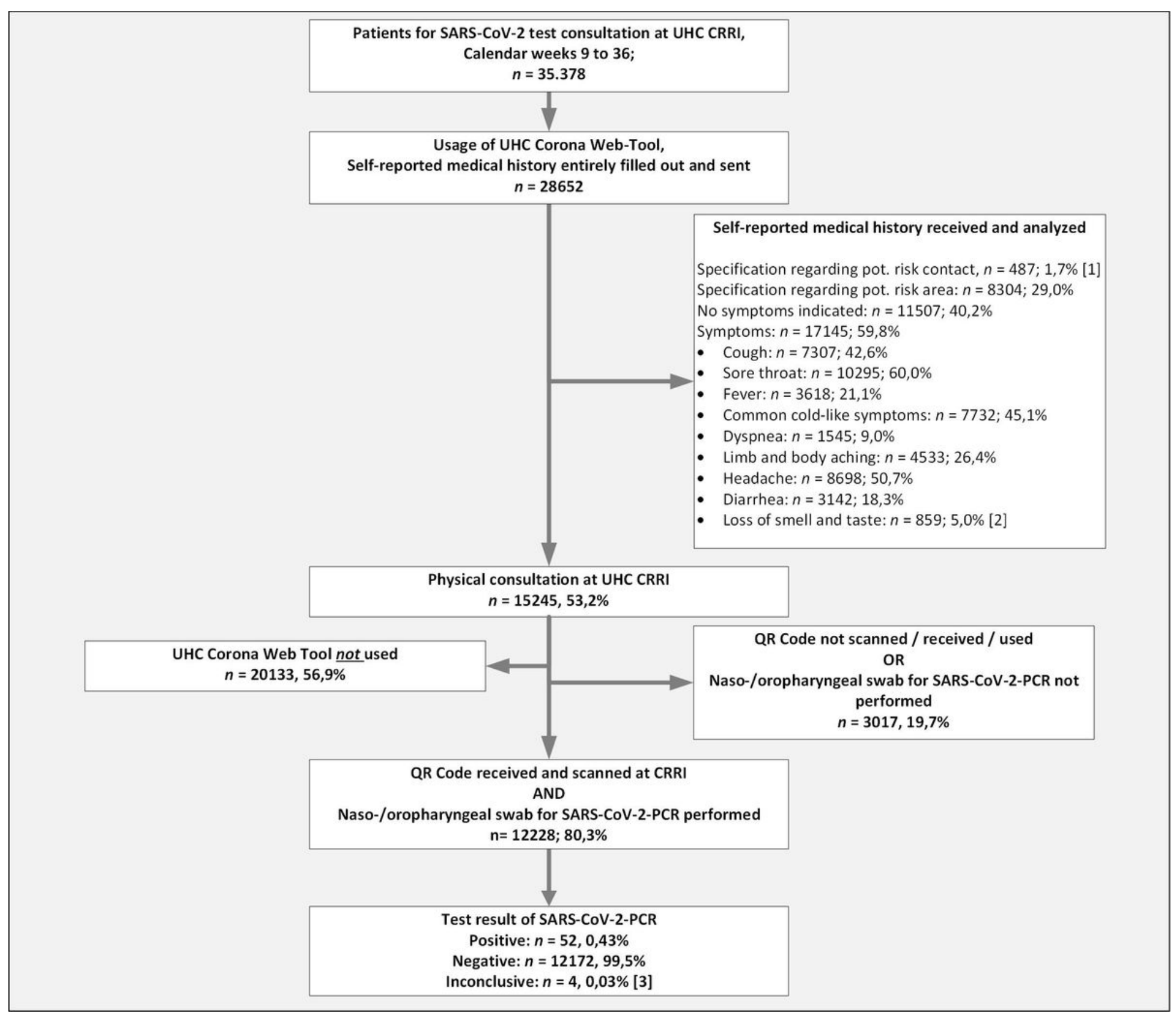

Figure 2

Characteristics of UHC Corona Web Tool users CRRI, COVID-19 rapid response infrastructure facility; UHC, University Hospital of Cologne [1] Feature excluded since calendar week 27 due to inaccurate answers [2] included since May 17th, 2020 (calendar week 28) [3] Inconclusive test results usually lead to a rapid notification of the patient and a repeated appointment for an additional swab for SARS-CoV-2 PCR; two patients did not consult the CRRI again after being notified to do so 


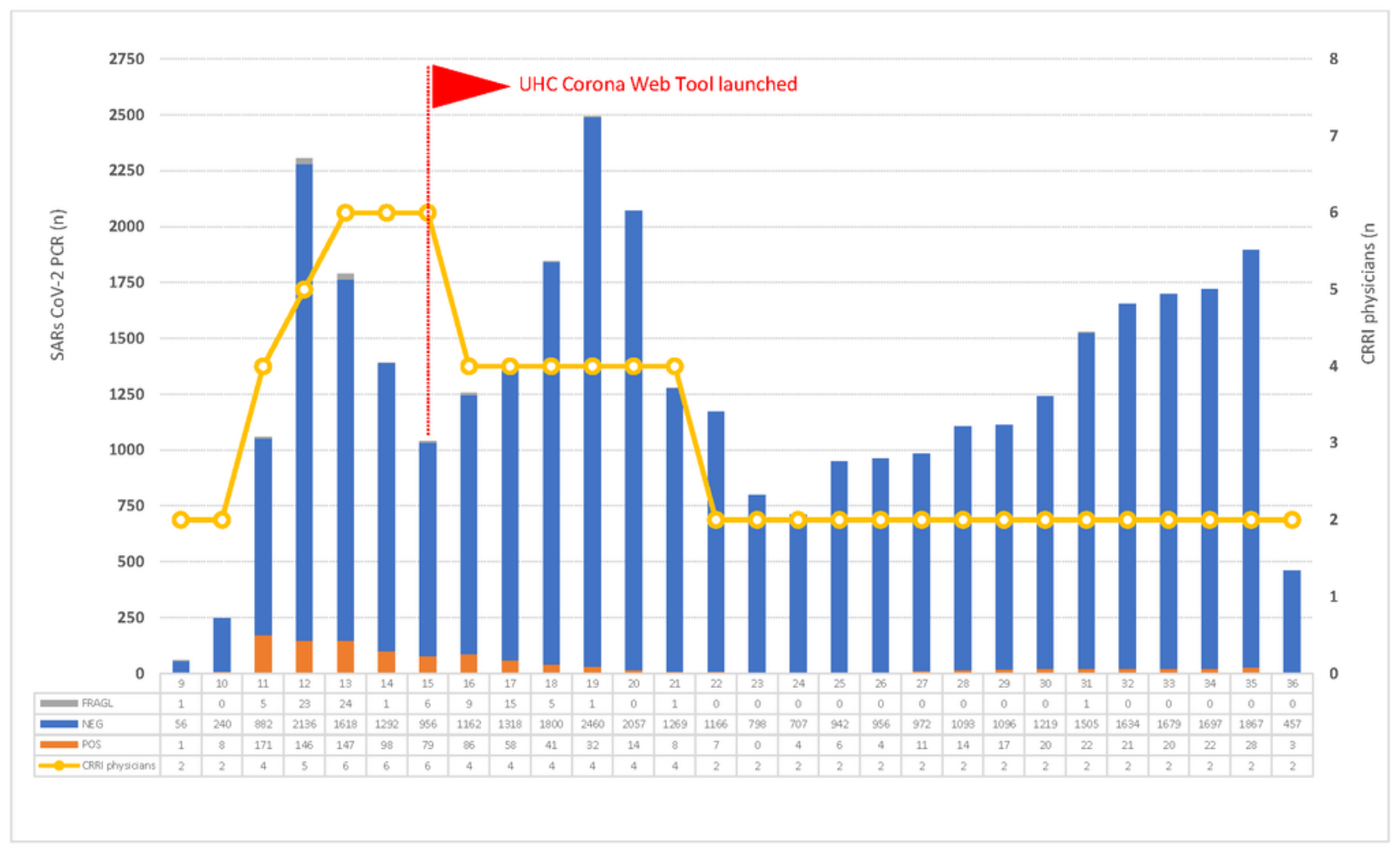

Figure 3

Patient contacts, number of UHC physicians and SARS-CoV-2 test results from naso-/oropharyngeal swabs at CRRI CRRI, COVID-19 rapid response infrastructure facility; UHC, University Hospital of Cologne, POS, positive SARS-CoV-2 test result; NEG, negative SARS-CoV-2 test result; FRAGL, inconclusive SARS-CoV-2 test result

\section{Supplementary Files}

This is a list of supplementary files associated with this preprint. Click to download.

- AdditionalFile1FigA1.pdf

- AdditionalFile2FigA2a.pdf

- AdditionalFile3FigA2b.pdf

- AdditionalFile4FigA2c.pdf

- AdditionalFile5FigA2d.docx 